\title{
Properties of the new boson
}

\author{
Fabio Cerutti, ${ }^{*}$ \\ Lawrence Berkeley National Laboratory \\ E-mail: fabio.cerutti@cern.ch \\ on behalf of the ATLAS, CMS, CDF and DO Collaborations.
}

\begin{abstract}
One year after the discovery claimed by the ATLAS and CMS Collaborations, supported by the measurements from the CDF and D0 Collaborations, the properties of the new boson have been studied with increasing accuracy using the LHC $p p$ collision data and the Tevatron $p \bar{p}$ data. The mass of the new resonance has been measured by ATLAS and CMS with a fit to the $H \rightarrow \gamma \gamma$ and $H \rightarrow Z Z^{*} \rightarrow 4 \ell$ mass spectra with an accuracy of a few per mill. The spin and parity have been probed by looking at observables sensitive to the $J^{P}$ quantum numbers of the new resonance and found to be in agreement with the Standard Model (SM) scalar hypothesis. The measurements of the production cross-sections times branching ratios are compatible with the SM Higgs boson predictions. The couplings of the new boson have been tested by performing a fit to the measured yields and found to be in agreement with SM predictions. In summary all measured properties are in agreement with the predictions of the Brout-Englert-Higgs $(\mathrm{BEH})$ mechanism for electroweak symmetry breaking.
\end{abstract}

The European Physical Society Conference on High Energy Physics -EPS-HEP2013

18-24 July 2013

Stockholm, Sweden

${ }^{*}$ Speaker. 


\section{Introduction}

The discovery of a new particle with mass of about $125 \mathrm{GeV}$ in the search for the Standard Model (SM) Higgs boson at the CERN Large Hadron Collider (LHC) [1], reported in July 2012 by the ATLAS [2] and CMS [3] Collaborations, is a milestone in the quest to understand the origin of electroweak symmetry breaking $[4,5,6,7,8,9]$. The present experimental challenge is to compare its properties with the SM predictions for the Higgs boson. In the SM, the Higgs boson is a spin-0 and CP-even particle $\left(J^{C P}=0^{+}\right)$. The Landau-Yang theorem forbids the direct decay of an onshell spin-1 particle into a pair of photons $[10,11]$. The spin-1 hypothesis is therefore strongly disfavored by the observation of the $H \rightarrow \gamma \gamma$ decay.

The CMS Collaboration has published a spin-parity study [12] based on the $H \rightarrow Z Z^{*}$ channel where the SM scalar hypothesis is favored over the pseudo-scalar hypothesis at a confidence level (CL) above 95\%. The ATLAS Collaboration has recently submitted two letters, based on the three main bosonic decay modes, on the measurement of the mass and couplings [13] and on the determination of the spin-parity quantum numbers [14]. The CDF and D0 Collaborations have recently submitted combined studies on the properties of the new boson [15].

At this conference an overview of the experimental searches for the SM Higgs boson has been presented in a dedicated talk [16]. In this document the combination of these searches and their interpretation in terms of the properties of the new boson are reported. In Section 2 the measurement of the mass of the new boson is presented. In Section 3 the SM scalar hypothesis is confronted with several alternative $J^{P}$ models, namely $J^{P}=0^{-}, 1^{+}, 1^{-}$and $2^{+}$. Studies on the production and decay rates and test of the couplings via fits to the data are reported in Section 4.

\section{Mass}

The measurement of the mass of the new boson is a crucial step in the determination of its properties. Once the mass is measured all cross-sections and branching ratios for the SM Higgs boson can be predicted with good accuracy.

The mass can be measured independent of assumptions on the couplings to the SM particles by a fit to the mass spectra of the channels with high mass resolution, namely the $H \rightarrow \gamma \gamma$ and $H \rightarrow Z Z^{*} \rightarrow 4 \ell$ decay modes.

The measured mass computed by combining these two channels by ATLAS [13] gives a value of $M_{H}=125.5 \pm 0.2_{\text {stat }} \pm 0.6_{\text {sys }} \mathrm{GeV}$ and by CMS [17] with a value of $M_{H}=125.7 \pm 0.3_{\text {stat }} \pm 0.3_{\text {sys }}$ $\mathrm{GeV}$. The two measurements are compatible with each other. In Figure 1 the test statistic used for the mass determination, minus twice the logarithm of the profiled likelihood, is plotted versus the boson mass for the two bosonic decay modes and their combination.

In addition CMS has also measured, by using the $H \rightarrow \gamma \gamma$ mass spectrum [18], the first direct constraint on the total width of the new boson $\Gamma_{H}<6.9 \mathrm{GeV}$ at $95 \% \mathrm{CL}$. This limit is far from the expected SM width of about $4 \mathrm{MeV}$ but is the first direct measurement of this important observable.

\section{Spin}

Several alternative models have been tested against the SM scalar hypothesis by the ATLAS, CMS and D0 Collaborations. The modeling of the alternative hypotheses is mainly based on 

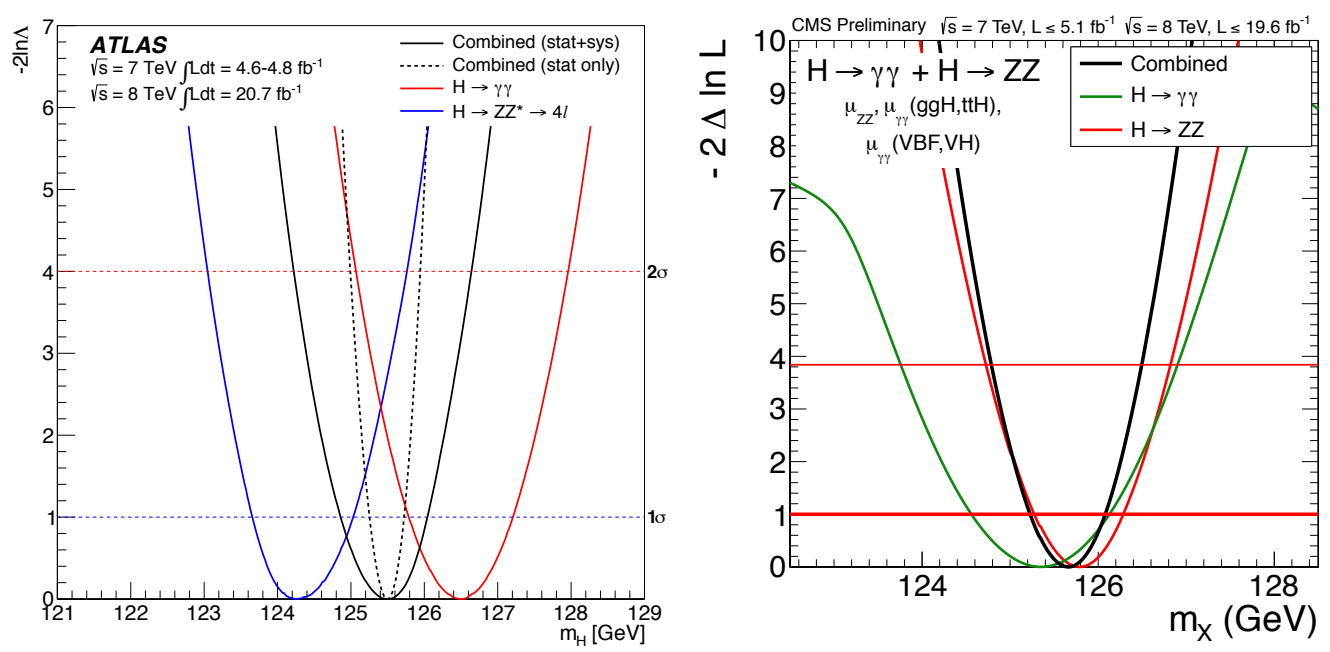

Figure 1: Values of the $-2 \log \left[L\left(M_{H}\right) / L\left(\hat{M}_{H}\right)\right]$ from the ATLAS (left) and CMS (right) Collaborations. The black curves show the combination of the two bosonic decay modes.

Ref. [19]. With this generator several alternative $J^{P}=0^{-}, 1^{+}, 1^{-}, 2^{+}$can be produced with leading order (LO) kinematics. Alternative generators are available, like the one described in $[20,21]$ that has been used by the D0 Collaboration to model the graviton-inspired $J^{P}=2^{+}$signal.

The CMS Collaboration has produced results mainly based on the $H \rightarrow Z Z^{*} \rightarrow 4 \ell$ final state [22]. In this final state several variables sensitive to the spin and parity of the new boson are available, namely two invariant masses and five angles. A summary of the CMS results can be found in Table 1. All non-scalar $J^{P}$ models are excluded at more then $95 \% \mathrm{CL}$ in favor of the SM hypothesis. For the $J^{P}=2^{+}$case CMS has also combined the $H \rightarrow W W^{*} \rightarrow \ell v \ell v$ and the $Z Z^{*}$ channels. For the $W W^{*}$ decay mode the most sensitive variables are the di-lepton azimuthal separation, $\Delta \phi_{\ell \ell}$ and the invariant mass $M_{\ell \ell}$. By combining these two channels the gluon-fusion produced $2^{+}$model is excluded at $99.5 \% \mathrm{CL}$.

\begin{tabular}{|l|c|c|c|c|c|c|}
\hline$J^{p}$ & production & comment & expect $(\mu=1)$ & obs. $0^{+}$ & obs. $J^{p}$ & CL $_{s}$ \\
\hline $0^{-}$ & $g g \rightarrow X$ & pseudoscalar & $2.6 \sigma(2.8 \sigma)$ & $0.5 \sigma$ & $3.3 \sigma$ & $0.16 \%$ \\
$0_{h}^{+}$ & $g g \rightarrow X$ & higher dim operators & $1.7 \sigma(1.8 \sigma)$ & $0.0 \sigma$ & $1.7 \sigma$ & $8.1 \%$ \\
$2^{+}{ }_{m g g}$ & $g g \rightarrow X$ & minimal couplings & $1.8 \sigma(1.9 \sigma)$ & $0.8 \sigma$ & $2.7 \sigma$ & $1.5 \%$ \\
$2^{+}{ }_{m} q \overline{\bar{q}}$ & $q \bar{q} \rightarrow X$ & minimal couplings & $1.7 \sigma(1.9 \sigma)$ & $1.8 \sigma$ & $4.0 \sigma$ & $<0.1 \%$ \\
$1^{-}$ & $q \bar{q} \rightarrow X$ & exotic vector & $2.8 \sigma(3.1 \sigma)$ & $1.4 \sigma$ & $>4.0 \sigma$ & $<0.1 \%$ \\
$1^{+}$ & $q \bar{q} \rightarrow X$ & exotic pseudovector & $2.3 \sigma(2.6 \sigma)$ & $1.7 \sigma$ & $>4.0 \sigma$ & $<0.1 \%$ \\
\hline
\end{tabular}

Table 1: Expected and observed rejection for different spin-parity models tested by the CMS Collaboration using the $H \rightarrow Z Z^{*} \rightarrow 4 \ell$ decay mode.

The CMS Collaboration has also studied in Ref. [22] the possibility to have a pseudo-scalar component in the $H \rightarrow Z Z^{*}$ decay. They have fitted the spin-sensitive variables of the $H \rightarrow Z Z^{*} \rightarrow$ $4 \ell$ decay mode for the fraction of the $\mathrm{CP}$-odd amplitude normalized to the sum of the SM plus the CP-odd ones, defined as $f_{a 3}=\left|A_{C P-o d d}\right|^{2} /\left(\left|A_{S M}\right|^{2}+\left|A_{C P-o d d}\right|^{2}\right)$. The fit to the data is compatible with the zero value of this fraction, giving an upper limit of $f_{a 3}<0.58$ at 95\% CL.

The ATLAS Collaboration has combined the results of the $H \rightarrow Z Z^{*} \rightarrow 4 \ell$ and $H \rightarrow W W^{*} \rightarrow \ell v \ell v$ 
channels when testing the spin-1 hypothesis while for the $2^{+}$model also the $H \rightarrow \gamma \gamma$ final state has been combined. In this last decay mode the variable sensitive to the spin is the decay angle $\left|\cos \theta^{*}\right|$ evaluated in Collins-Soper frame [23]. These studies have been published by ATLAS in Ref. [14]. The observed and expected $\mathrm{CL}_{\mathrm{S}}$ values for the exclusion of the alternative spin-parity hypotheses are summarized in Figure 2.

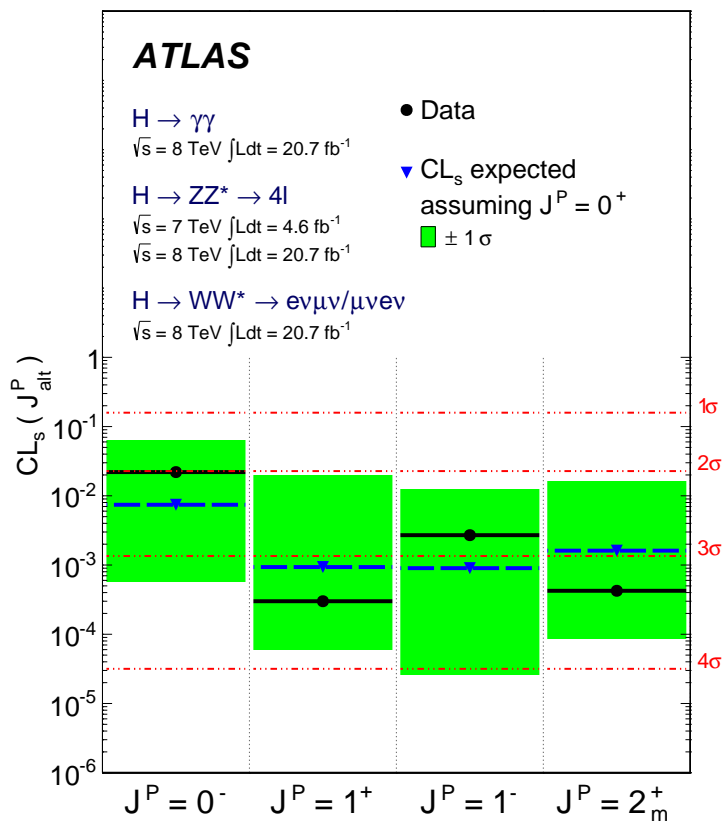

Figure 2: Expected (blue triangles/dashed lines) and observed (black circles/solid lines) confidence level $\mathrm{CL}_{\mathrm{s}}$ for alternative spin-parity hypotheses assuming a SM $J^{P}=0^{+}$signal. The green band represents the $68 \% \mathrm{CL}_{\mathrm{s}}\left(J_{\text {alt }}^{P}\right)$ expected exclusion range for a signal with assumed $J^{P}=0^{+}$. On the right $y$-axis, the corresponding numbers of Gaussian standard deviations are given, using the one-sided convention.

At this conference the D0 Collaboration has presented for the first time an analysis based on the $V H \rightarrow V b b$ decay mode [24]. This analysis aimed to distinguish a graviton-like signal, $J^{P}=2^{+}$, from the SM one. The production of a tensor is characterized by a $\beta^{5}$ threshold behavior of the $V H$ system to be confronted with a $\beta$ behavior for the SM scalar. This implies that variables like the invariant mass of the $Z H \rightarrow \ell \ell b b$ system or the transverse mass of the $W H \rightarrow \nu \ell b b$ system are expected to be strongly sensitive to the spin nature of the new boson. This is presented in Figure 3 where the mass of the $\mathrm{ZH} \rightarrow \ell \ell b b$ system with the D0 data is shown. Using this kind of variables, combining the $\ell v b b, v v b b$ and $\ell \ell b b$ final states the $2^{+}$model is excluded at more than $99.9 \% \mathrm{CL}$ assuming a signal strength of $\mu=1.23$ (that corresponds to the one measured by the main SM search from the D0 Collaboration).

In summary all alternative spin-parity hypotheses tested have been rejected at more than $95 \%$ CL while the SM scalar hypothesis gives a good description of the experimental data. However, a small $\mathrm{CP}$-odd component in the new boson couplings cannot be excluded by these studies and represents the next experimental challenge. 


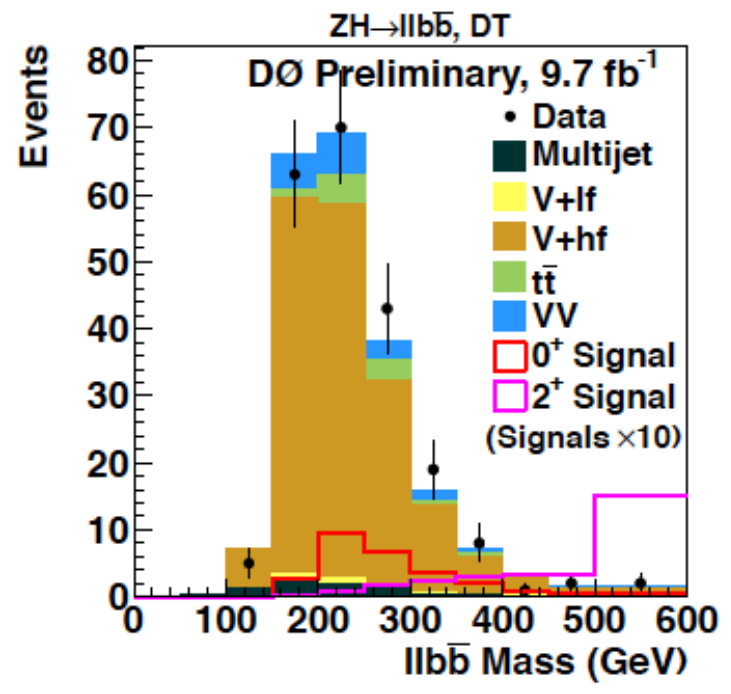

Figure 3: Distribution of the $\ell \ell b b$ invariant mass from the D0 Collaboration for the data (back dots), the SM backgrounds (full colored histograms) and signal (red histogram) and for the $2^{+}$model (magenta histogram).

\section{Rates}

The measurement of the production and decay rates of the newly discovered boson is a crucial test to understand whether it corresponds to the SM Higgs boson responsible for the EW symmetry breaking.

At the Tevatron the main production mode is via associated $V H$ production and the main decay mode is to the $b b$ final state. In addition the CDF and D0 searches also include the $\gamma \gamma, W W^{*}$ and $\tau \tau$ final states [15].

At the LHC four different production mechanisms are available for the SM Higgs, the most abundant one is gluon-fusion, followed by vector-boson fusion (VBF), by the $\mathrm{W}$ or $\mathrm{Z}$ associated production $(\mathrm{VH})$ and by the $t \bar{t}$ associated production $(\mathrm{ttH})$. Several final states $\left(\gamma \gamma, \tau \tau, b \bar{b}, W W^{*}\right.$, $\left.Z Z^{*}, Z \gamma, \mu \mu\right)$ are expected to be experimentally accessible, once final center-of-mass energy and luminosity will be achieved by the LHC collider.

The ATLAS and CMS analyses [13, 17] are designed to give sensitivity to different production modes for the different final states. Dedicated categories with selections based on lepton(s), transverse momentum imbalance and jets are designed to distinguish the $\mathrm{VBF}, \mathrm{VH}$ and ttH production modes from the most abundant gluon-fusion one.

The most accurate test of the couplings of the new boson is the combined signal strength. The signal strength is defined, for a given channel, as the measured value of the signal yield normalized to the SM Higgs boson expectation $\mu=(\sigma \times B R) /(\sigma \times B R)_{S M}$. This normalized quantity can be combined between different production and decay modes. The combined signal strengths from ATLAS, CMS and Tevatron (CDF and D0 combined) are shown in Figure 4. The values of the combined signal strengths are: $1.33 \pm 0.20$ from ATLAS, $0.80 \pm 0.14$ from CMS and $1.44 \pm 0.60$ from CDF and D0 combined. For all different decay modes, the signal strengths are compatible with the SM expectation of unity, with the largest deviation at the 1.9 standard deviations level for 
the ATLAS $\gamma \gamma$ decay mode.
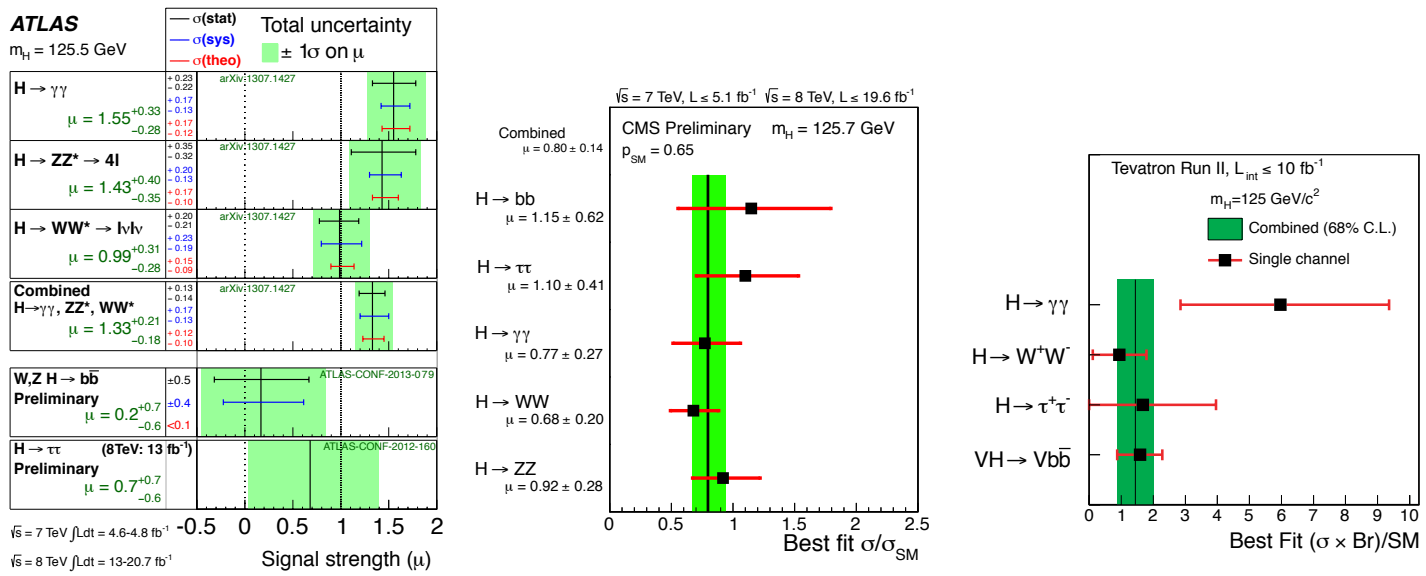

Figure 4: Signal strengths from the ATLAS (left), CMS (center) and combined CDF-D0 (right) Collaborations for the main decay modes and their combinations.

An interesting question that can be asked is whether we can claim the observation of vectorboson mediated production. This question has been addressed by ATLAS and CMS combining the results of several decay modes with categories designed to distinguish the VH and VBF production modes from the dominant gluon-fusion production. The observed yields are fitted in terms of the ratio between the $\mathrm{VH}+\mathrm{VBF}$ signal strengths and the the gluon-fusion $(\mathrm{gg})+\mathrm{ttH}$ ones (expected to be mediated by fermions in the SM). In this ratio, for each channel, the dependency on the decay BR cancels out. The CMS result for this ratio is shown in Figure 5 in terms of minus twice the profiled likelihood ratio. The zero value of the ratio $\mu_{V B F+V H} / \mu_{g g+t t H}$ is disfavored at more than 3 standard deviations. A similar analysis performed by ATLAS is shown in Figure 5. In this case the the logarithm of the profiled likelihood ratio is shown as a function of $\mu_{V B F} / \mu_{g g+t t H}$, with the VH signal strength profiled to the data (i.e., fixed to the value that maximize the profile likelihood for each value of the parameter of interest). Also in this case the zero value for the VBF signal strength is disfavored at more than 3 standard deviations.

In summary both CMS and ATLAS Collaborations have presented results that give evidence for vector-mediated production at more than 3 standard deviations.

Another interesting question is whether the new boson couples to fermions. The combined $\mathrm{CDF}$ and D0 results [25] for the $V H \rightarrow V b b$ final state provided first evidence for direct fermion couplings (and also for vector-boson mediated production) at 2.8 standard deviations for an Higgs boson mass of $125 \mathrm{GeV}$. The CMS Collaboration has recently combined the signal strengths for the $H \rightarrow b b$ and $H \rightarrow \tau \tau$ decay modes. This combination is incompatible with the absence of these decay modes at 3.4 standard deviations, hence providing evidence for direct couplings to the fermions.

In the framework of the SM the couplings of the new boson can be tested with high accuracy. In order to benefit from the correlation between production and decay modes the deviation from SM coupling predictions are parametrized via coupling scale factors $\kappa$ 's. The SM predictions are confirmed if all fitted $\kappa$ 's are compatible with the value of one. The details of this parametrization can be found in Ref. [26]. With current statistics only a limited number of parametrizations can be 

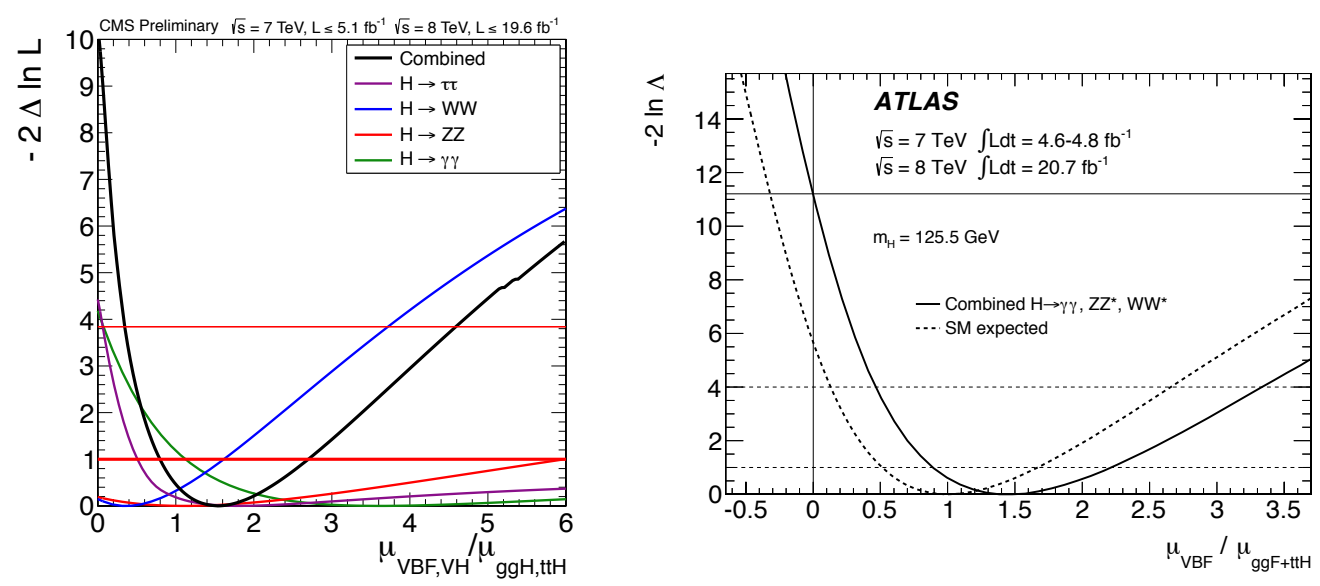

Figure 5: Left: value of the $-2 \Delta \log (L)$ as a function of the ratio $\mu_{V B F+V H} / \mu_{g g+t t H}$ from the CMS Collaboration. Right: value of the $-2 \Delta \log (L)$ as a function of the ratio $\mu_{V B F} / \mu_{g g+t t H}$ from the ATLAS Collaboration.

fitted to the data. Several sectors of the new boson couplings have been tested by the ATLAS and CMS Collaborations and by the CDF and D0 Collaborations combined. Namely the couplings to the vector-boson and fermionic sectors, the ratio of the couplings to the $W$ and $Z$ (related to the custodial symmetry), the loop-induced couplings to gluons and photons, the ratio of couplings to up and down-type fermions, the beyond SM (BSM) contributions to the total width. A summary of these tests, under the assumptions described in Refs. [13, 17], is shown in Figure 6.
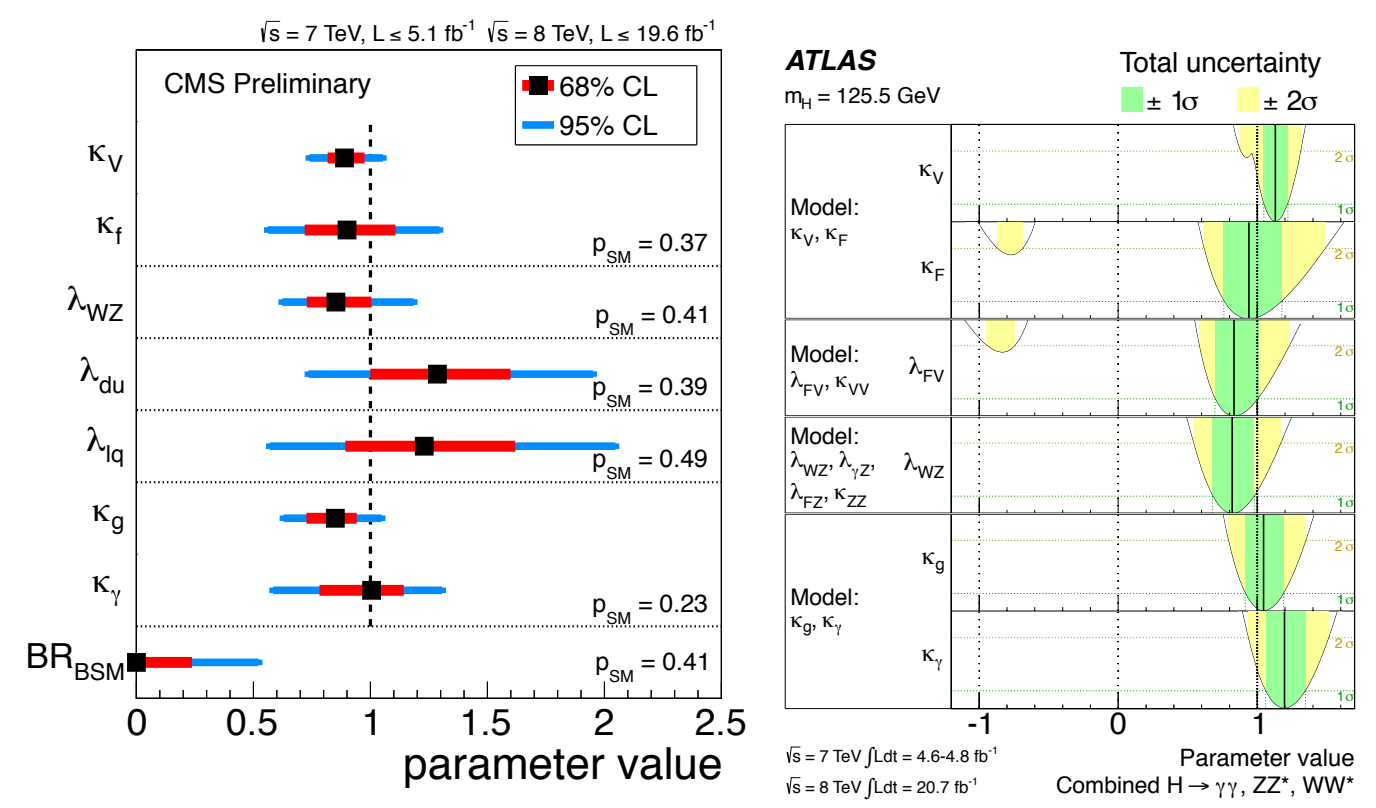

Figure 6: Summary of several coupling tests performed by the CMS (left) and ATLAS (right) Collaborations.

All tested models are compatible with the SM Higgs boson expectations, with probabilities above the $12 \%$ level. 
In summary the studies of the measured yields give results compatible with the SM expectations and show evidence for vector-boson production and direct couplings to fermions.

\section{Conclusions}

One year after its discovery the properties of the new boson have been studied with increasing accuracy thanks the the analysis of LHC Run1 data from the ATLAS and CMS Collaborations and of the full Tevatron data from the CDF and D0 Collaborations.

The mass of the new boson is measured with a few per mill accuracy from the $H \rightarrow \gamma \gamma$ and $H \rightarrow Z Z^{*} \rightarrow 4 \ell$ mass spectra by ATLAS and CMS giving compatible results.

The spin and parity are in agreement with the SM scalar hypothesis while several alternative spin-parity models, namely $J^{P}=0^{-}, 1^{+}, 1^{-}, 2^{+}$, are rejected at more then $95 \% \mathrm{CL}$.

The measured yields in several production and decay modes are in agreement with the SM Higgs boson predictions. All tests on the couplings performed on LHC and Tevatron data give results compatible with the SM expectations.

In summary all measured properties are in agreement with the predictions of the Brout-EnglertHiggs (BEH) mechanism for electro-weak symmetry breaking. The experimental challenge now is to look for (small) deviations of its properties from the SM Higgs predictions that could hint at the presence of physics BSM in the Higgs sector.

\section{Acknowledgements}

I want to warmly thank the organizers of the conference for the wonderful organization and the beatiful atmosphere in which this conference was held. I also wish to thanks my ATLAS, CMS, $\mathrm{CDF}$ and D0 colleagues for the great help and support received in preparing this talk.

\section{References}

[1] L. Evans and P. Bryant, LHC Machine, JINST 3 no. 08, (2008) S08001.

[2] ATLAS Collaboration, Observation of a new particle in the search for the Standard Model Higgs boson with the ATLAS detector at the LHC, Phys. Lett. B 716 (2012) 1, arXiv: 1207.7214 [hep-ex].

[3] CMS Collaboration, Observation of a new boson at a mass of $125 \mathrm{GeV}$ with the CMS experiment at the LHC, Phys. Lett. B 716 (2012) 30, arXiv:1207.7235 [hep-ex] .

[4] F. Englert and R. Brout, Broken symmetry and the mass of gauge vector mesons, Phys. Rev. Lett. 13 (1964) 321.

[5] P. W. Higgs, Broken symmetries, massless particles and gauge fields, Phys. Lett. 12 (1964) 132.

[6] P. W. Higgs, Broken symmetries and the masses of gauge bosons, Phys. Rev. Lett. 13 (1964) 508.

[7] G. S. Guralnik, C. R. Hagen, and T. W. B. Kibble, Global conservation laws and massless particles, Phys. Rev. Lett. 13 (1964) 585.

[8] P. W. Higgs, Spontaneous symmetry breakdown without massless bosons, Phys. Rev. 145 (1966) 1156.

[9] T. W. B. Kibble, Symmetry breaking in non-Abelian gauge theories, Phys. Rev. 155 (1967) 1554. 
[10] L. D. Landau, On the angular momentum of a two-photon system, Dokl. Akad. Nauk Ser. Fiz. 60 (1948) 207.

[11] C.-N. Yang, Selection Rules for the Dematerialization of a Particle Into Two Photons, Phys. Rev. 77 (1950) 242.

[12] CMS Collaboration, Study of the mass and spin-parity of the Higgs boson candidate via its decays to Z boson pairs, Phys. Rev. Lett. 110, arXiv:1212.6639 [hep-ex].

[13] ATLAS Collaboration, Measurements of Higgs boson production and couplings in diboson final states with the ATLAS detector at the LHC, submitted to Phys. Lett. B (2013).

[14] ATLAS Collaboration, Evidence for the spin-zero nature of the Higgs boson using ATLAS data, submitted to Phys. Lett. B (2013).

[15] TEVNPH (Tevatron New Phenomena and Higgs Working Group), CDF and D0 Collaborations, Higgs boson studies at Tevatron, Accepted by Phys. Rev. D (2013), arXiv:1303.6346 [hep-ex] .

[16] G. Landsberg, Higgs bosons in the Standard Model and beyond, EPS-2103 Stocholm, this Conference Proceedings (2013).

[17] CMS Collaboration, Measurements of the properties of the new boson with a mass near $125 \mathrm{GeV}$, CMS-PAS-Hig-2013-005 (2013).

[18] CMS Collaboration, Properties of the observed Higgs-like resonance decaying into two photons, CMS-PAS-Hig-2013-016 (2013).

[19] Y. Gao et al., Spin determination of single-produced resonances at hadron colliders, Phys. Rev. D 81 (2010) 075022, arXiv:1001.3396 [hep-ph] .

[20] P. de Aquino, K. Hagiwara, Q. Li, and F. Maltoni, Simulating graviton production at hadron colliders, JHEP 1106 (2011) 132, arXiv:1101.5499 [hep-ph].

[21] J. Alwall et al., MadGraph 5 : Going Beyond, JHEP 06 (2011) 128, arXiv: 1106.0522.

[22] CMS Collaboration, Properties of the Higgs-like boson in the decay $H \rightarrow Z Z^{*} \rightarrow 4 \ell$ in pp collisions at $\sqrt{s}=7$ and $8 \mathrm{TeV}$, CMS-PAS-Hig-2013-012 (2013).

[23] J. C. Collins and D. E. Soper, Angular distribution of dileptons in high-energy hadron collisions, Phys. Rev. D 16 (1977) 2219-2225.

[24] D0 Collaboration, Constraints on the $J^{P}=2^{+}$hypothesis for the $125 \mathrm{GeV}$ boson in W/Z + bb final states, D0 note CONF-6387 (2013).

[25] D0 and CDF Collaborations, Evidence for a particle produced in association with weak bosons and decaying to a bottom-antibottom quark pair in Higgs boson searches at the Tevatron, Phys. Rev. Lett. 109 (2012), arXiv:1207.6436 [hep-ex] .

[26] LHC Higgs Cross Section Working Group, S. Heinemeyer, C. Mariotti, G. Passarino, and R. Tanaka (Eds.), Handbook of LHC Higgs Cross Sections: 3. Higgs Properties, arXiv:1307.1347 [hep-ph]. 